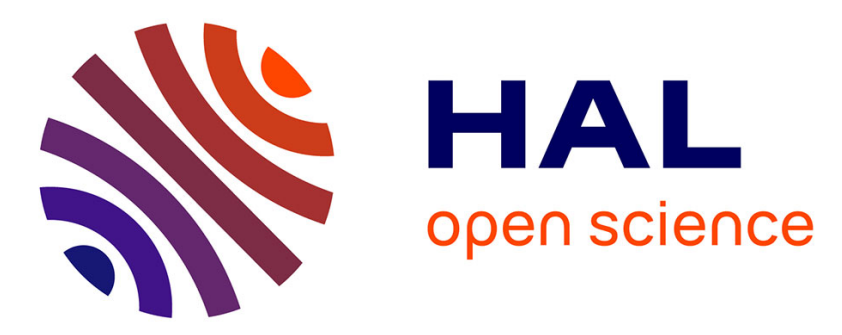

\title{
Characterization technique of optical whispering gallery mode resonators in the microwave frequency domain for optoelectronic oscillators
}

\author{
Pierre-Henri Merrer, Khaldoun Saleh, Olivier Llopis, Simone Berneschi, \\ Franco Cosi, G. Nunzi Conti
}

\section{To cite this version:}

Pierre-Henri Merrer, Khaldoun Saleh, Olivier Llopis, Simone Berneschi, Franco Cosi, et al.. Characterization technique of optical whispering gallery mode resonators in the microwave frequency domain for optoelectronic oscillators. Applied optics, 2012, 51 (20), pp.4742-4748. hal-00798034

\section{HAL Id: hal-00798034 \\ https://hal.science/hal-00798034}

Submitted on 7 Mar 2013

HAL is a multi-disciplinary open access archive for the deposit and dissemination of scientific research documents, whether they are published or not. The documents may come from teaching and research institutions in France or abroad, or from public or private research centers.
L'archive ouverte pluridisciplinaire HAL, est destinée au dépôt et à la diffusion de documents scientifiques de niveau recherche, publiés ou non, émanant des établissements d'enseignement et de recherche français ou étrangers, des laboratoires publics ou privés. 


\title{
Characterization technique of optical whispering gallery
}

\author{
mode resonators in the microwave frequency domain for
}

\section{optoelectronic oscillators}

\author{
Pierre-Henri Merrer, ${ }^{1,2, *}$ Khaldoun Saleh, ${ }^{1,2,3}$ Olivier Llopis, ${ }^{1,2}$ S. Berneschi, ${ }^{4,5}$ F. Cosi, ${ }^{5}$ and \\ G. Nunzi Conti ${ }^{5}$ \\ ${ }^{1}$ CNRS, LAAS, 7 avenue du colonel Roche, F-31400 Toulouse, France \\ ${ }^{2}$ Univ de Toulouse, LAAS, F-31400 Toulouse, France \\ ${ }^{3}$ CNES, 18 avenue Edouard Belin, F-31401 Toulouse, France \\ ${ }^{4}$ Centro Studi e Ricerche “Enrico Fermi”, Piazza del Viminale 2, 00184 Rome, Italy \\ ${ }^{5}$ CNR-IFAC, "NelloCarrara” Institute of Applied Physics, Via Madonna del Piano 10, 50019 \\ Sesto Fiorentino, Firenze, Italy \\ "Corresponding author:phmerrer@laas.fr
}

Optical Q factors measurements are performed on a Whispering Gallery Mode (WGM) disk resonator using a microwave frequency domain approach instead of using an optical domain approach. An absence of hysteretic behavior and a better linearity are obtained when performing linewidth measurements by using a microwave modulation for scanning the resonances instead of the piezoelectric based frequency tuning capability of the laser. The WGM resonator is then used to stabilize a microwave optoelectronic oscillator. The microwave output of this system generates a $12.48 \mathrm{GHz}$ signal with $-94 \mathrm{dBc} / \mathrm{Hz}$ phase noise at $10 \mathrm{kHz}$ offset. 
OCIS codes: $230.5750,230.4910,350.4010$.

\section{Introduction}

Investigations towards high spectral purity sources are a very important and interesting point in the microwave field, concerning many applications ranging from communications and radar to time-frequency metrology. The need for microwave sources able to provide high frequency (10 to $100 \mathrm{GHz}$ ), versatility of the output frequency and low phase noise signal is growing in response to advances in technology related to optical communication links, requiring an efficient high-data-rate system, and radar systems, requiring the detection of weak signals from a dense background clutter. Two classical approaches can be distinguished for high spectral microwaves sources: the use of quartz or SAW (surface acoustic wave) oscillators at low frequencies, which can be multiplied up to the desired frequency, or, the use of dielectric resonator oscillators, which includes ceramic and monocrystalline resonators (mainly sapphire) [1]. In the first case, the multiplication entails a degradation of the phase noise of $20 \log \mathrm{N}$, with $\mathrm{N}$ being the multiplication factor. In the other case, the main problem with ceramic resonator is that its quality factor $(\mathrm{Q})$ is inversely proportional to the frequency. In the special case of sapphire resonator, the disadvantage is its size because it is based on high order modes. Finally, the technologies for high spectral purity microwave sources are reaching their limits when the goal is to get simultaneously high performance in term of phase noise, high operation frequency and small size, especially in embedded systems.

A solution to overcome these limitations is to carry the microwave signal on another wave, such as an acoustic wave or an optical wave. The case of the acoustic wave is today investigated in thin film bulk acoustic wave resonators. This approach allows obtaining a small size about $100 \mu \mathrm{m}^{2}$, which focus the wireless market [2]. However these resonators are limited in 
their operating frequency (up to $5 \mathrm{GHz}$ ) and their $\mathrm{Q}$ factor values (in the range of 500). The case of optics is different: what we are looking for is very high performance at higher frequencies. The operation principle uses an optical carrier, delivered by a laser, a modulator, to transpose the microwave signal around the optical carrier, an optical frequency reference device (resonator or delay line) and a fast photodiode to bring back the signal to microwaves. It is today a well known technique, already commercialized, and this type of oscillator is known as an optical-electronic oscillator (OEO). The first OEO has been proposed in 1994 [3]. This type of source is based on an optical delay line, and features today ultra low phase noise performance (ex: $-163 \mathrm{dBc} / \mathrm{Hz}$ at $10 \mathrm{kHz}$ offset of a $10 \mathrm{GHz}$ carrier [4]). However, these oscillators are quite bulky because of the fiber spool and their phase noise spectrum features many parasitic modes due to the large number of frequencies for which a $2 \pi$ phase condition is realized in these devices. Some improvements of this approach, based on the coupling of an optical oscillator and a microwave oscillator (COEO), have demonstrated again the great potential of this approach to reach very low phase noise levels [5].

An alternative approach can be to replace the optical fiber delay line by a whispering gallery mode (WGM) optical resonator [6]. These resonators can be fabricated with crystalline material, such as calcium fluoride $\left(\mathrm{CaF}_{2}\right)$, to achieve extremely high Qs [7]. Further improvement of OEO parameters can be achieved with this solution. Firstly, the size of the optical resonator is quite small, because of the small wavelength of the optical signal. Thus, the microwave signal carried by the optical carrier can be trapped in a resonator much smaller than the microwave wavelength. In this case, the microwave oscillation frequency is defined by the free-spectral-range (FSR) of the WGM resonator. Moreover, in opposition to the case of dielectric resonators, the equivalent microwave $\mathrm{Q}$ factor increases with the microwave 
frequency. This technology appears to be a disruptive technology in the field of microwave sources.

In this paper, we report on a characterization method for high-Q optical whispering gallery mode disk resonators in the microwave domain. This approach allows a measurement of the resonance characteristics of these resonators with a very good linearity. Moreover, the measured data is obtained in the same way the resonators are used afterwards in an OEO system, and the measured Q factor is the OEO Q factor. We briefly describe the polishing procedure of crystalline disks and the coupling method in order to trap laser light into the ultra-low loss WGMs. We then present in detail the experimental setup and the resonator characterization procedure. Finally, we present the experimental results on phase noise measurement of the OEO.

\section{Fabrication of WGM resonator and coupling method}

Special equipment was developed for manufacturing optical resonators based on calcium fluoride disks (about $5.5 \mathrm{~mm}$ in diameter). These disk-resonators were made from commercial $0.5 \mathrm{~mm}$ thick $\mathrm{CaF}_{2}$ windows by core drilling a cylinder and thereafter polishing the edge into a spheroidal shape. We have optimized the polishing procedure of crystalline disks using a homemade lapping station, which is shown in Fig.1. The almost spherical profile is obtained through a rotational stage whose pivot point can be finely adjusted. The polishing protocol is divided in six successive steps starting with an initial two-step grinding phase (disks with abrasive size of 12 and 9 microns) which is followed by a four-step finer polishing phase, based on polycrystalline diamond suspensions with decreasing grit sizes $(3,1,0.25$, and 0.05 microns, respectively). After each step a rather critical cleaning procedure using organic solvents has been implemented.

Concerning the coupling method, several techniques have been investigated in order to obtain a selective and efficient coupling into the WGM resonator. The oldest method and the 
common technique is the prism coupling configuration [8]. This approach can be compacted using an angle polished optical fiber [9]. We have chosen here an alternative method that enables high efficiency coupling directly from optical fiber where it is possible to obtain the total transfer of the incoming power to the WGM [10]. Indeed, tapered optical fibers meet the key requirements for a good performance and easy coupling: phase matching and sufficient overlap between the evanescent electromagnetic fields. Phase-matching is accomplished by matching the taper propagation constant to the WGM's propagation constant while the overall coupling strength varies exponentially with the air gap between the resonator and the taper. In practice, these coupling conditions can be optimized by varying the position of the resonator along the biconical fiber taper (which has a variable waist diameter along its length), and by controlling the air gap distance. The minimum taper diameter in our work is typically less than $1 \mu \mathrm{m}$, with loss levels of less than $1 \mathrm{~dB}$. They are made by heating a single-mode silica fiber (typically SMF-28) with a torch and slowly pulling the fiber. Phase matching is possible because $\mathrm{CaF}_{2}$ and silica have similar refractive indices at $1550 \mathrm{~nm}$. A dedicated measurement bench has thus been set up, using three-axis piezoelectric nanometric stage (P-611 Nanocube XYZ piezo stage) to accurately position the tapers with respect to the optical resonator. The tapers are kept with a slight tension and the measurement bench has been built on an optical table in order to minimize the influence of vibrations. This allows us to get an efficient dual-coupling with tapered fibers. A schematic of the coupling system is show in Fig. 2. Two nearly identical taper couplers are placed tangentially at the opposite sides of the disk. One serves as the input coupler, and the other as the output coupler.

\section{Resonator characterization}


The first step in OEO studies is to set up a characterization bench for the resonators to be used in the OEO. It is essential to test the resonator behavior in a configuration which is as close as possible to the OEO system. The microwave characterization bench is depicted in Fig. 3. The test configuration, which has been developed was clearly dedicated to OEO design. In general the light coupled inside the WGM resonator induces a power dependent thermal shift of the mode frequency leading to thermal instability. It was demonstrated $[11,12]$ that the frequency drift due to the resonator self heating can be used for thermal locking of the laser frequency to the WGM. This technique is not applicable in the case of $Q$-factor measurement, which must not be perturbed by thermal nonlinearity. Thus, a more robust frequency locking mechanism has been set up using a Pound Drever Hall (PDH) feedback loop [13 - 15]. The validity of this approach has been verified experimentally on a different type of optical resonator featuring similar $\mathrm{Q}$ factor: the fiber ring resonator [16]. As soon as the laser is locked onto the resonance, it is possible to use the system for microwave applications. The laser used in this configuration is a fiber laser (Koheras Adjustik) with a very narrow linewidth $(1 \mathrm{kHz})$. A microwave optical intensity modulator (Mach-Zehnder modulator, MZM) is added to the system and we use an allfiber polarization controller in order to select TE or TM modes. The signal coming out from a microwave network analyzer (Anritsu 37347A) feeds the modulator, goes through the optical resonator and is finally recovered on a fast photodiode and analyzed on port 2 of the vectorial network analyzer (VNA). The bandwidth of the MZM and the photodiode is around $20 \mathrm{GHz}$, which is larger than the FSR of the WGM resonator. Fig. 4 represents the MZM frequency response measured with the VNA. The bandwidth is defined as the $-3 \mathrm{~dB}$ high cut-off frequency of the $S_{21}$ curve. The power deviation of the $S_{21}$ curve can be taken into account by doing a calibration of VNA, in order to obtain a linear fit of the MZM frequency response in the 
frequency range $5 \mathrm{GHz}$ to $20 \mathrm{GHz}$. The WGM Q factor can then be measured as the laser carrier is locked onto one of the resonances while the microwave source of the VNA is scanning the WGM resonances with the laser sidebands in the MZM bandwidth. The relative frequency difference between the optical carrier and its sidebands is precisely defined by the microwave source which is accurately controlled in frequency. We therefore get the resonance profile of the WGMs in the microwave domain, instead of a direct measurement in the optical domain. An absence of hysteretic behavior is obtained on these measurements thanks to the MZM biased close to its linear modulation voltage $\left(V_{D C}=V_{\frac{\pi}{2}}\right)$ and the linear fit of the MZM frequency response is obtained by the calibration of the VNA. The most common optical method used to characterize high-quality resonators, based on a slow scan of the wavelength of a narrow probe laser, which is obtained from a piezoelectric device (acting either directly on the grating of DFB fiber lasers or on the mirror of external cavity semiconductor laser) [17, 18], cannot achieve this linearity. Fig. 5 shows the hysteretic behavior of our laser output wavelength vs piezoelectric component voltage. At a fixed wavelength we encounter different slopes depending on the scanning direction. Additionally same non linearity may be also observed in the same graph. On the contrary the only requirement of the microwave approach is to able to lock the laser frequency close to the center of the resonance frequency. Fig. 6a shows an example of such a measurement from $5 \mathrm{GHz}$ to $20 \mathrm{GHz}$ performed on our $\mathrm{CaF}_{2}$ disk at the through port, when the laser carrier is locked to the fundamental WGM. Fig. $6 \mathrm{~b}$ focuses on this mode and Fig. $6 \mathrm{c}$ gives the information on its resonance half bandwidth $(\delta v)$ and $\mathrm{Q}$ factor $(\mathrm{Q}=v / \delta v)$ at the drop port. The measured Q factor at the drop port is about $8.4 \times 10^{7}$.

\section{Optoelectronic oscillator}


As previously stated, one of the basic issues in the realization a WGM-based OEO is the locking of the optical carrier onto one of the fundamental WGM in order to avoid thermal nonlinearities. The same approach using a low frequency PDH feedback loop may be applied to stabilize the laser on a WGM resonance. A microwave feedback loop is then used to start an oscillation on one of the lateral side-modes of the resonator. This high frequency loop contains amplifiers, attenuators and a phase shifter in order to adjust the conditions for the oscillation start up. The OEO set-up is depicted in Fig. 7. The power at the $\mathrm{CaF}_{2}$ disk entrance is about $4.5 \mathrm{dBm}$. The coupling with tapered optical fiber is rather efficient ( $3 \mathrm{~dB}$ insertion losses) so the power at the drop port is $1.5 \mathrm{dBm}$. A $3 \mathrm{~dB}$ optical coupler is placed before the photodiode in order to perform the phase noise measurement and the power on the photodiode is $\mathrm{Pm}=-2.5 \mathrm{dBm}$ (phase noise measurement is performed in a $20 \mathrm{~m}$ remote location, and the signal is brought to the signal analyser through optical fiber). The high frequency loop includes two RF amplifiers: the first one with a gain of $34 \mathrm{~dB}$ and the second one with a gain of $25 \mathrm{~dB}$. A $10 \mathrm{~dB}$ RF splitter allows to measure the spectrum of the microwave signal generated in the OEO and an attenuator with $11 \mathrm{~dB}$ attenuation is placed after the first amplifier. The RF power at the photodiode output in operating OEO is about $10 \mu \mathrm{W}$ and the power at the input of the MZM is $19.5 \mathrm{dBm}$. The oscillation features $8 \mathrm{dBm}$ output power and is obtained with $48 \mathrm{~dB} \mathrm{RF}$ amplification. As theoretically predicted [19], the system has an oscillation frequency equal to the resonator FSR $(12.481 \mathrm{GHz})$.

Fig. 8 shows a typical phase noise spectrum of the $\mathrm{OEO}$ referenced on a $\mathrm{CaF}_{2}$ disk. This phase noise has been measured for a frequency offset, $\mathrm{f}_{\mathrm{m}}$, ranging from $10 \mathrm{~Hz}$ to $100 \mathrm{MHz}$. The phase noise spectrum features different slopes. In the first one, between $10 \mathrm{~Hz}$ and $10 \mathrm{kHz}$, the noise slope of $-30 \mathrm{~dB} / \mathrm{dec}$ corresponds to an important $1 / \mathrm{f}$ frequency noise contribution. This $1 / \mathrm{f}$ 
frequency noise may be due to some optical noise conversion processes in the oscillating system, or to the laser PDH stabilization loop, or of another source of 1/f noise such as the 1/f noise of amplifiers or of the photodiode. The white frequency noise is characterized by a slope of $-20 \mathrm{~dB} / \mathrm{dec}$ from $10 \mathrm{kHz}$ to $700 \mathrm{kHz}$, and it is linked to the Q-factor and to the noise to carrier ratio (NCR) of the optical part of the system. The phase noise floor appears after $700 \mathrm{kHz}$, and features a value of about $-133 \mathrm{dBc} / \mathrm{Hz}$.

The expected phase noise of the OEO, or at least the contributions other than the one of the $1 / \mathrm{f}$ noise, can be calculated using a simplified model of the OEO, following Leeson's approach [20]. Such a model leads to the following equation (1), which depends on the experimental parameters such as the microwave equivalent quality factor $\mathrm{Q}$, the measured optical power $\mathrm{P}_{\mathrm{m}}$, the sensitivity of the photodiode $\mathrm{R}(\lambda)$, the modulation index $\mathrm{m}$, the microwave frequency $v$, the offset frequency $f_{m}$ and the different noise contributions to the NCR.

$$
L\left(f_{m}\right)=20 \log \left(\frac{v}{2 \sqrt{2} Q f_{m}}\right)+10 \log \left(\frac{2\left(S_{R I N}+S_{t h}+S_{\text {shot }}\right)}{P_{m}^{2} R(\lambda)^{2} m^{2}}\right)
$$

with the thermal noise being $S_{\text {th }}(2)$, the shot noise $S_{\text {shot }}(3)$ and the laser noise $S_{\text {RIN }}(4)$ :

$$
\begin{aligned}
& S_{t h}=\frac{4 k_{B} T}{R}(2) \\
& S_{\text {shot }}=2 q R(\lambda) P_{m}(3) \\
& S_{R I N}=P_{m}^{2} R(\lambda)^{2} R I N
\end{aligned}
$$

where $\mathrm{T}$ is the room temperature, $\mathrm{k}_{\mathrm{B}}$ is the Boltzmann constant, $\mathrm{q}$ is the electron charge, $\mathrm{RIN}$ is the relative intensity noise of the laser and $\mathrm{R}$ is the photodiode load resistance. This model is of course only valid in the frequency range on which the phase noise is dominated by the white frequency or phase noise contribution (additive noise effect). Knowing in our setup that 
$\mathrm{P}_{\mathrm{m}}=-2.5 \mathrm{dBm}, \mathrm{R}(\lambda)=0.8 \mathrm{~A} / \mathrm{W}, \mathrm{m}=0.8, \mathrm{R}=50 \Omega$, and $\mathrm{RIN}=-148 \mathrm{dBc} / \mathrm{Hz}$, we can compare the simulated white phase noise with the experimental results (see Fig. 8)

Finally, the phase noise performance is evaluated to be $-94 \mathrm{dBc} / \mathrm{Hz}$ at $10 \mathrm{kHz}$ from the carrier, and $-116 \mathrm{dBc} / \mathrm{Hz}$ at $100 \mathrm{kHz}$. This phase noise level is better than the one recently demonstrated in [21], even though the oscillator generating a $10.7 \mathrm{GHz}$ signal was based on a resonator having a similar $Q$ factor (however the two OEOs topologies are very different). This performance is close to the one of commercially available microwave dielectric resonator oscillators (DROs). However, the increase in Q factor of the optical resonator by 10 to 100 times would result in a reduction by 20 to $40 \mathrm{~dB}$ in the OEO phase noise, and such ultra high Qs have already been demonstrated in this type of resonators, although DRO technology is an old technology which is close to its limits in performance.

\section{Conclusion}

Optical resonators allow reaching really high quality factors in the microwave range. This technology overcomes some limitations of conventional microwave technology for the development of high spectral purity microwave sources. In this paper, an accurate microwave characterization technique dedicated to optical high $\mathrm{Q}$ resonators has been reported. This method allows assessing resonators optical Q factor from linewidth measurements by using a microwave modulation for scanning the resonances instead of an optical frequency modulation. An improved linearity and absence of hysteretic behavior are obtained with this approach. A X-band microwave source using a $\mathrm{CaF}_{2}$ disk resonator and efficient dual-coupling with tapered fibers has then been realized. Even if the measured phase noise performance of this OEO is still modest, Even if the measured phase noise performance of this OEO is still modest, this performance will 
certainly improve in the future thanks to a better understanding of the noise processes (specially the $1 /$ f noise) and an improvement of the resonator technology.

This work was supported by the funding from ANR (National Research Agency) project “ORA” 2010 BLAN 031203.

\section{References}

1. G. Cibiel, M. Regis, O. Llopis, A. Rennane, L. Bary, R. Plana, Y. Kersale, and V. Giordano, "Optimization of an ultra low-phase noise sapphire-SiGe HBT oscillator using nonlinear CAD," IEEE Trans. Ultrason., Ferroelectr., Freq. Control 51, 33-41 (2004).

2. C. Campbell, "Surface acoustic wave devices for mobile and wireless communications" (Academic Press, 1998).

3. X. S. Yao and L. Maleki, "High frequency optical subcarrier generator," Electronics Letters 30, 1525-1526 (1994).

4. D. Eliyahu, D. Seidel, and L. Maleki, "RF Amplitude and phase-noise reduction of an optical link and an opto-electronic oscillator," IEEE Trans. Microw. Theory Tech. 56, 449-456 (2008).

5. D. Eliyahu and L. Maleki, "Modulation response (S21) of the coupled opto-electronic oscillator," in Proceedings of IEEE Conference on Frequency Control Symposium, (IEEE, 2005), 850-856.

6. A. A. Savchenkov, V. S. Ilchenko, J. Byrd, W. Liang, D. Eliyahu, A. B. Matsko, D. Seidel, and L. Maleki, "Whispering-gallery mode based opto-electronic oscillators," in Proceedings of IEEE Conference Frequency Control Symposium, (IEEE, 2010), 554-557. 
7. A. A. Savchenkov, A. B. Matsko, V. S. Ilchenko, and L. Maleki, "Optical resonators with ten million finesse," Opt. Express 15, 6768-6773 (2007).

8. V. B. Braginsky, M. L. Gorodetsky, and V. S. Ilchenko, "Quality-factor and nonlinear properties of optical whispering-gallery modes," Physics Letters A 137, 393-397 (1989).

9. V. S. Ilchenko, X. S. Yao, and L. Maleki, "Pigtailing the high-Q microsphere cavity: a simple fiber coupler for optical whispering-gallery modes," Opt. Lett. 24, 723-725 (1999).

10. M. Cai and K. Vahala, "Highly efficient optical power transfer to whispering-gallery modes by use of a symmetrical dual-coupling configuration," Opt. Lett. 25, 260-262 (2000).

11. T. Carmon, L. Yang, and K. Vahala, "Dynamical thermal behavior and thermal self-stability of microcavities," Opt. Express 12, 4742-4750 (2004).

12. P. Del'Haye, O. Arcizet, A. Schliesser, R. Holzwarth, and T. J. Kippenberg, "Full stabilization of a microresonator-based optical frequency comb," Phys. Rev. Lett. 101, 053903 (2008).

13. R. W. P. Drever, J. L. Hall, F. V. Kowalski, J. Hough, G. M. Ford, A. J. Munley, and H. Ward, "Laser phase and frequency stabilization using an optical resonator," Appl. Phys. B, 31, 97-105 (1983).

14. T. Carmon, T.J. Kippenberg, Y. Lan, R. Hosein, S. Sean and K.J. Vahala, "Feedback control of ultra-high-Q microcavities: application to micro-Raman lasers and microparametric oscillators, " Opt. Express 13, 3558-3566 (2005)

15. D. O'Shea, A. Rettenmaier and A. Rauschenbeutel, "Active frequency stabilization of an ultra-high Q whispering-gallery-mode microresonator," Applied Physics B, 4, 623-627 (2010) 
16. P.-H. Merrer, O. Llopis, and G. Cibiel, "Laser stabilization on a fiber ring resonator and application to RF filtering," IEEE Photon. Technol. Lett. 20, 1399-1401 (2008).

17. C. Schmidt, A. Chipouline, T. Pertsch, A. Tünnermann, O. Egorov, F. Lederer, and L. Deych, "Nonlinear thermal effects in optical microspheres at different wavelength sweeping speeds," Opt. Express 16, 6285-6301 (2008).

18. P.-H. Merrer, A. Bouchier, H. Brahimi, O. Llopis, and G. Cibiel, "High-Q optical resonators for laser stabilization in microwave photonics oscillators," in Proceedings of IEEE Conference on CLEO Europe, (IEEE 2009), 1-1.

19. A. B. Matsko and V. S. Ilchenko, "Optical resonators with whispering-gallery modes-part I: basics," IEEE Journal of Selected Topics in Quantum Electronics 12, 3-14 (2006).

20. D. B. Leeson, "A simple model of feedback oscillator noise spectrum," Proceedings of the IEEE 54, 329-330 (1966).

21. K. Volyanskiy, P. Salzenstein, H. Tavernier, M. Pogurmirskiy, Y. K. Chembo, and L. Larger, "Compact optoelectronic microwave oscillators using ultra-high Q whispering gallery mode disk-resonators and phase modulation," Opt. Express 18, 22358-22363 (2010).

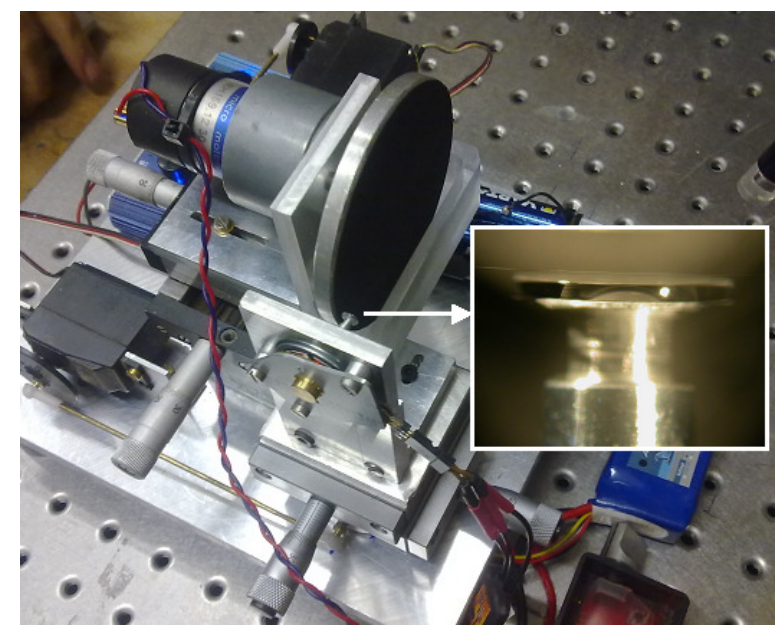


Fig.1: (Color online) Monocrystalline disk polishing station with the CaF2 disk magnified in the inset.

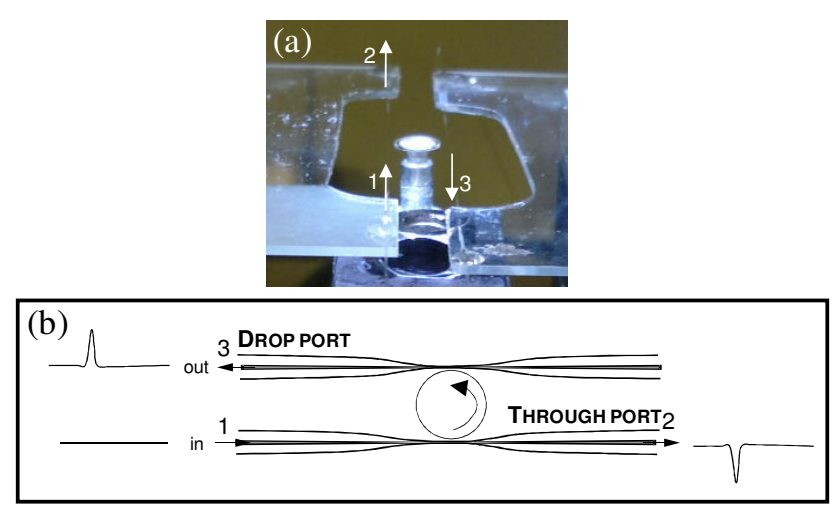

Fig.2. (Color online) Picture (a) and scheme (b) of the dual-coupling system with tapered fibers symmetrically coupled to the $\mathrm{CaF}_{2}$ disk.

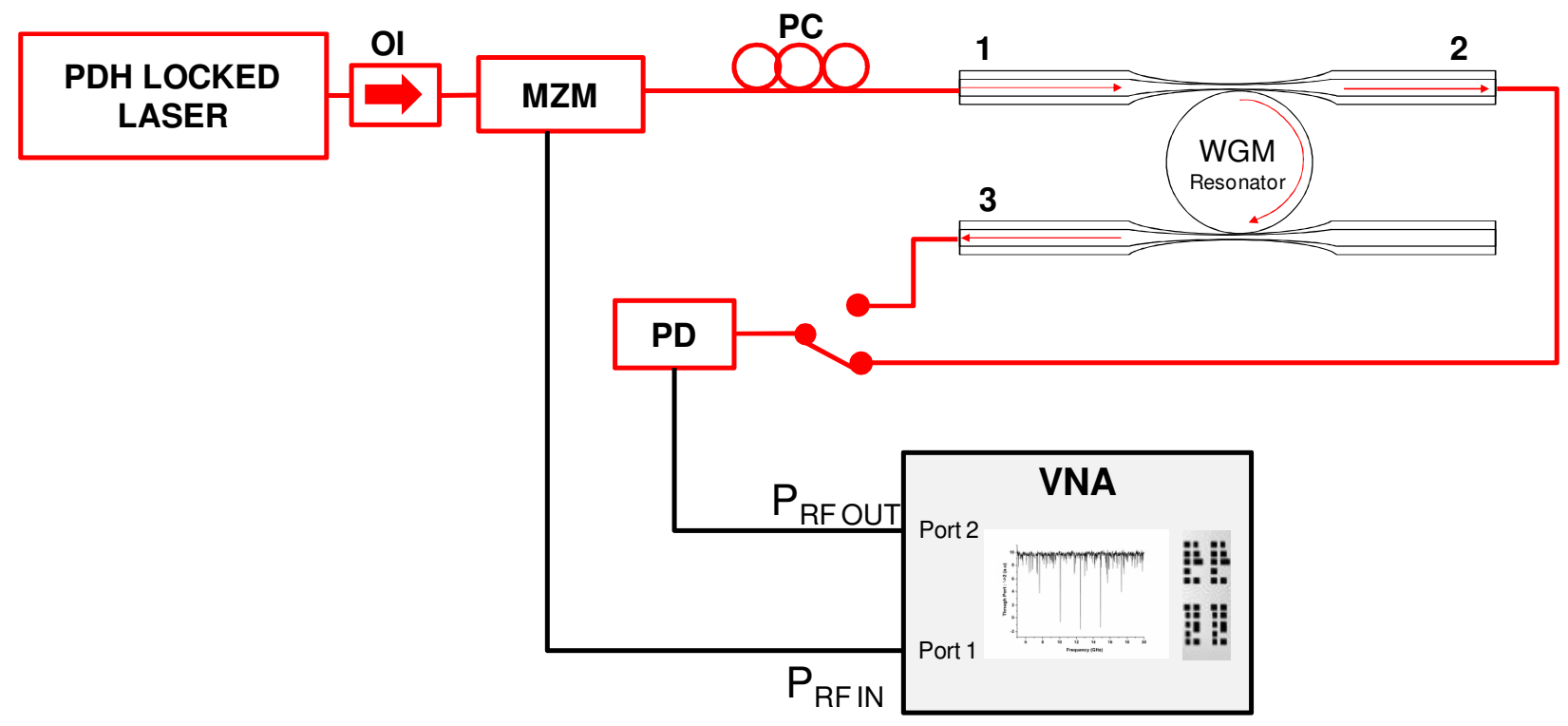

Fig.3. (Color online) Experimental setup of the microwave characterization technique used to measure the Q factor of high-quality resonators. OI : Optical Isolator, MZM : Mach-Zehnder 
Modulator, PC : Polarization Controller, PD : Photodiode, VNA : Vector Network Analyzer. (This figure should not be reduced)

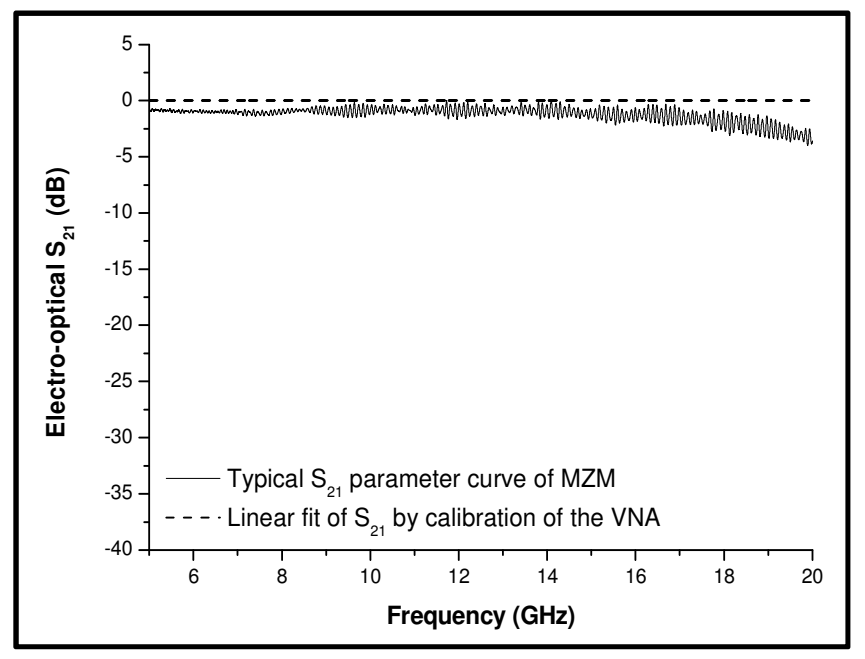

Fig.4. MZM frequency response obtained with $40 \mathrm{GHz}$ Network Analyzer. The measured bandwidth is around $20 \mathrm{GHz}$ which is larger than the FSR of the $\mathrm{Caf}_{2}$ disk. The linear fit of the frequency response $S_{21}$ is obtained by a calibration of the VNA.

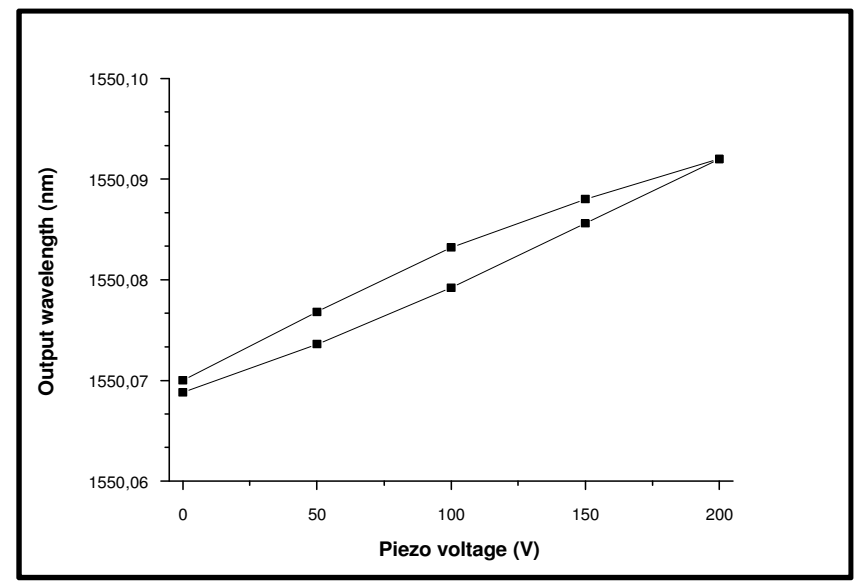

Fig.5. Hysteretic behavior of our Koheras Adjustik laser tuning mechanism based on a piezoelectric component. 


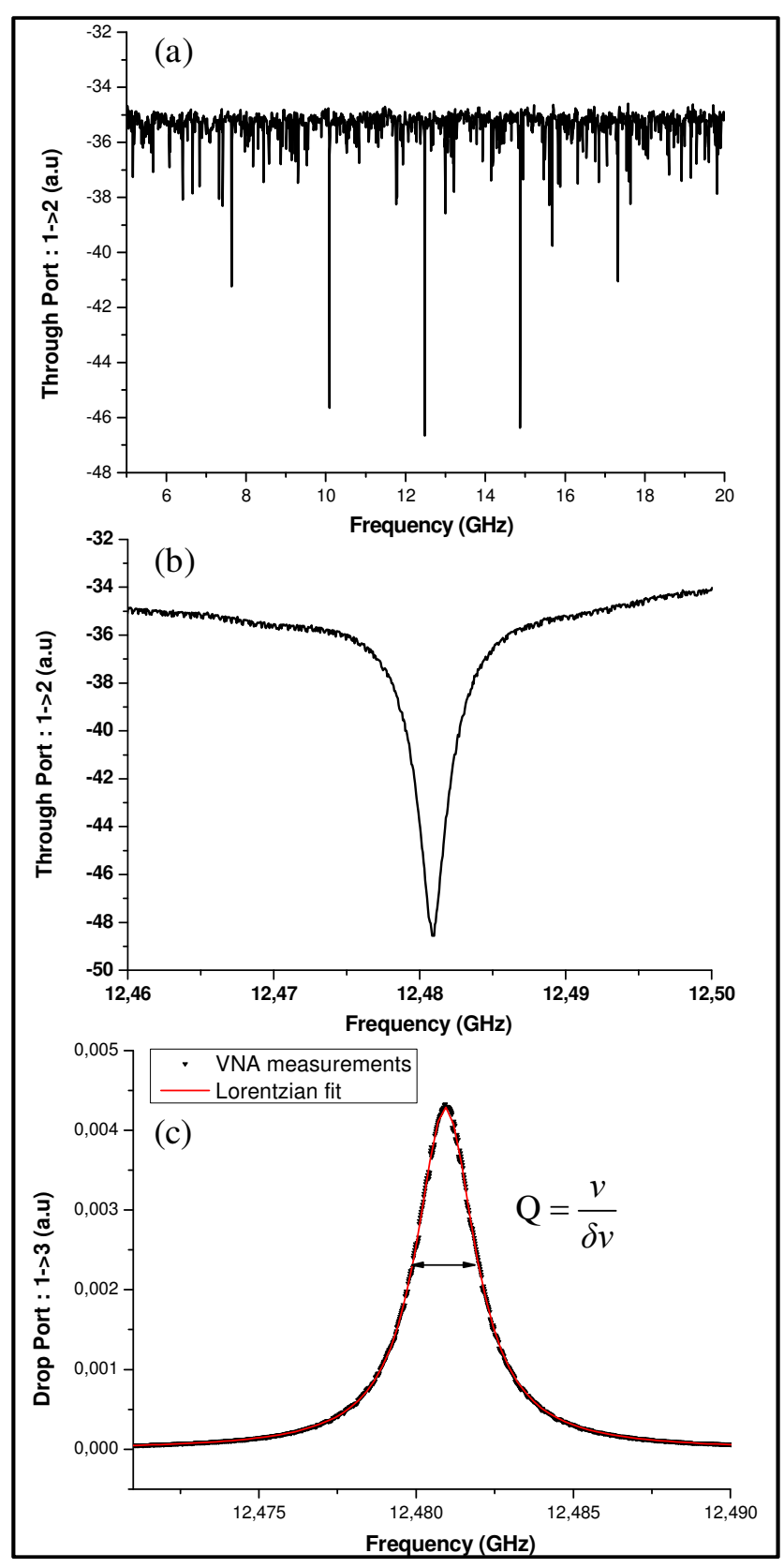

Fig.6. (a) (Color online) Measured spectrum of $\mathrm{a} \mathrm{CaF}_{2}$ disk at the through port using the microwave approach. (b) A focus on the fundamental mode. (c) Main resonance at the drop port, which gives a $Q$-factor of $8.4 \times 10^{7}$. 


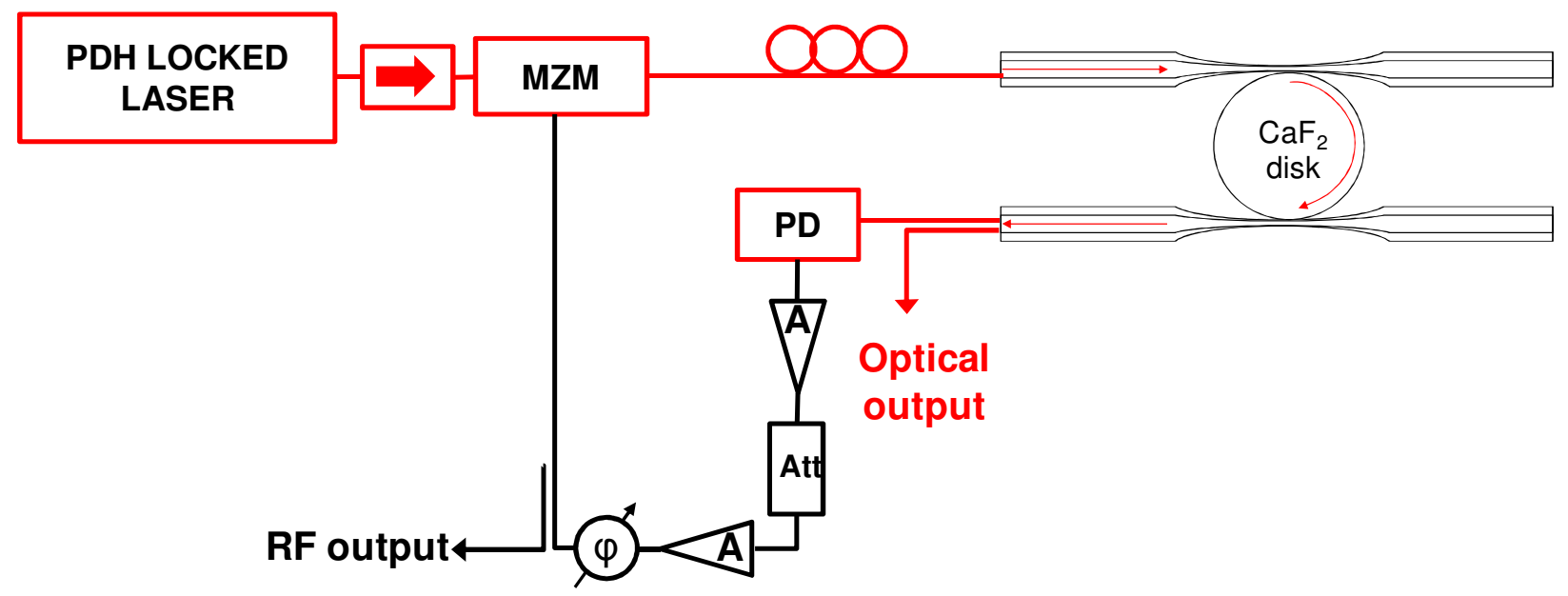

Fig. 7. (Color online) Experimental setup of the WGM-based OEO. A : microwave amplifier, Att : microwave attenuator. (This figure should not be reduced)

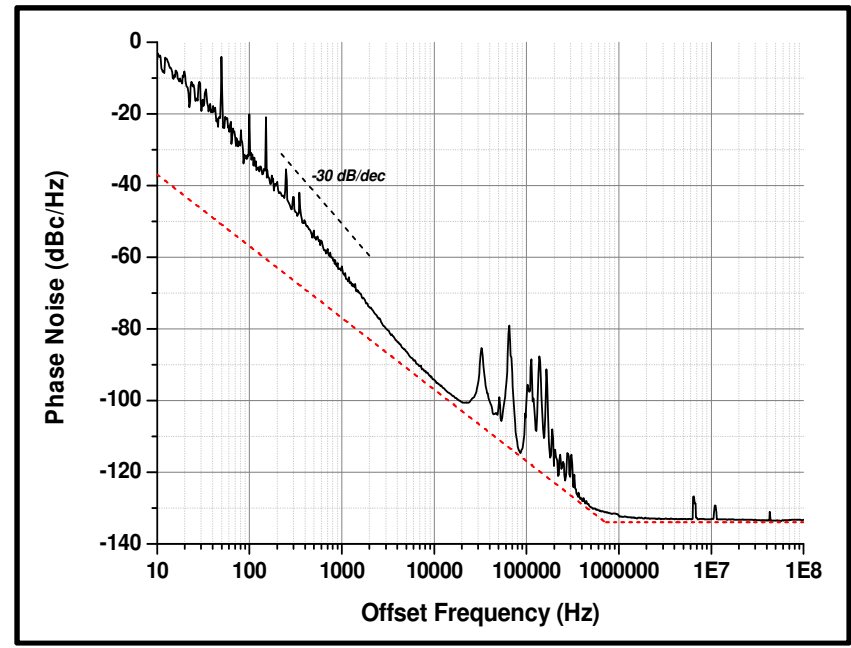

Fig.8. (Color online) Simulated phase noise spectrum (dash line) for the white frequency noise contribution area and experimental result (solid line). Measurement performed on an Agilent E5052B signal source analyzer. 